This is the final peer-reviewed accepted manuscript of:

F. Zonzini, M. Zauli, M. Mangia, N. Testoni and L. De Marchi, "Model-assisted Compressed Sensing for Vibration-based Structural Health Monitoring," in IEEE Transactions on Industrial Informatics, doi: 10.1109/TII.2021.3050146.

The final published version is available online at:

https://doi.org/10.1109/TII.2021.3050146

Rights / License:

The terms and conditions for the reuse of this version of the manuscript are specified in the publishing policy. For all terms of use and more information see the publisher's website. 


\title{
Model-assisted Compressed Sensing for Vibration-based Structural Health Monitoring
}

\author{
Federica Zonzini, Student Member, IEEE, Matteo Zauli, Student Member, IEEE, Mauro Mangia, Member, IEEE, \\ Nicola Testoni, Member, IEEE, and Luca De Marchi, Member, IEEE
}

\begin{abstract}
The main challenge in the implementation of longlasting vibration monitoring systems is to tackle the constantly evolving complexity of modern 'mesoscale' structures. Thus, the design of energy aware solutions is promoted for the joint optimization of data sampling rates, on-board storage requirements, and communication data payloads.

In this context, the present work explores the feasibility of the rakeness-based compressed sensing (Rak-CS) approach to tune the sensing mechanism on the second-order statistics of measured data. In particular, a novel model-assisted variant (MRak-CS) is proposed, which is built on a synthetic derivation of the spectral profile of the structure by pivoting on numerical priors. Moreover, a signal-adapted sparsity basis relying on the Wavelet Packet Transform operator is conceived, which aims at maximizing the signal sparsity while allowing for a precise timefrequency localization.

The adopted solutions were tested with experiments performed on a sensorized pinned-pinned steel beam. Results prove that the rakeness-based compression strategies are superior to conventional eigenvalue approaches and to standard CS methods. The achieved compression ratio is equal to 7 and the quality of the reconstructed structural parameters is preserved even in presence of defective configurations.
\end{abstract}

Index Terms-Compressed Sensing, Model-assisted Rakeness, Operational Modal Analysis, Structural Health Monitoring, Wavelet Packet Transform

\section{INTRODUCTION}

$\mathbf{T}$ HE evolving complexity of current Structural Health Monitoring (SHM) scenarios introduces new challenges for the integrity assessment of mesoscale structures [1], i.e. structures with very complex and wide geometries such as wind turbines, bridges, aircraft. Indeed, thanks to the recent advancements in Structural Mechanics, increasingly larger and more complicated infrastructures are built, implying the adoption of novel monitoring technologies basing on the most advanced research of the information and communication engineering community [2]. In particular, real-time, long-term

F. Zonzini, M. Zauli and N. Testoni, are with the Advanced Research Center on Electronic Systems for Information and Communication Technologies "Ercole De Castro", ARCES, University of Bologna, 40136 Bologna, Italy (e-mail: federica.zonzini@unibo.it; matteo.zauli7@unibo.it; nicola.testoni@unibo.it).

M. Mangia, L. De Marchi are with the Department of Electrical, Electronic and Information Engineering, DEI, University of Bologna, 40136 Bologna, Italy (e-mail: mauro.mangia2@unibo.it; 1.demarchi@unibo.it).

Manuscript received ; revised. This work has been funded by INAIL within the BRIC/2018, ID = 11 framework, project MAC4PRO ("Smart maintenance of industrial plants and civil structures via innovative monitoring technologies and prognostic approaches") and in part by the European Union's Horizon 2020 Research and Innovation Program under Arrowhead Tools Project under Grant Agreement 826452.

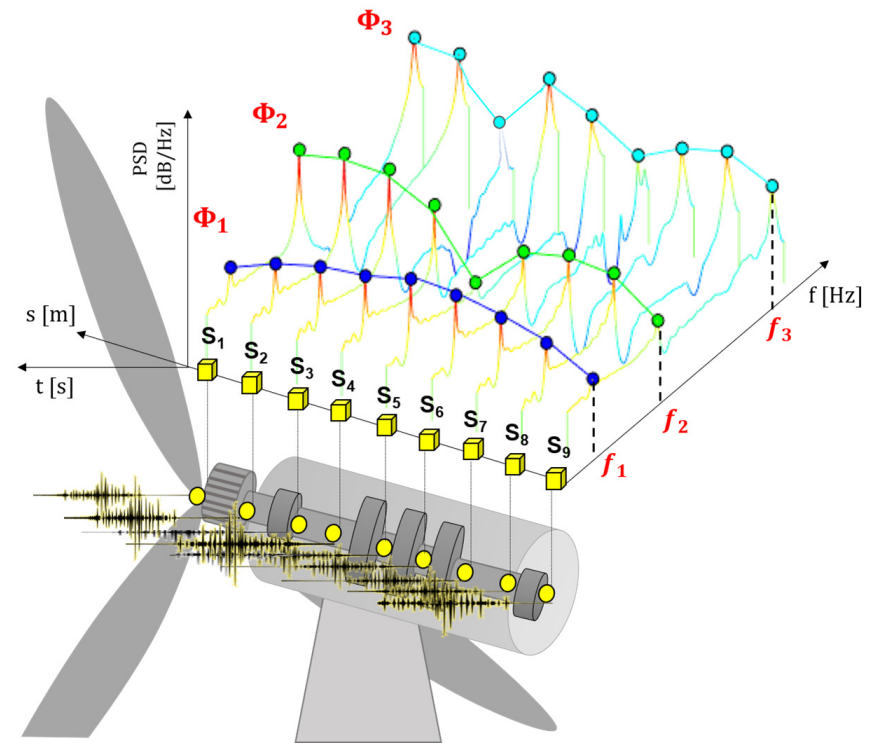

Fig. 1. Example of OMA-based processing flow and extracted modal parameters (in red fonts): natural frequencies $\left(f_{1}, f_{2}, f_{3},\right)$ correspond to the frequency peaks in the spectral domain, whereas the absolute value of the mode shapes $\left(\left|\Phi_{1}\right|,\left|\Phi_{2}\right|,\left|\Phi_{3}\right|\right)$ can be computed by interpolating in spatial domain the spectral peak values associated with each sensing position at a given natural frequency.

and low-power functionalities are primary issues to be tackled while designing a reliable and cost-effective SHM system [3].

It is worth noting that the spectral representation of vibration data is sparse. This characteristic makes the Compressed Sensing (CS) paradigm [4] applicable in this application domain. CS approaches are based on the adoption of sensing matrices which project data onto lower-dimensional signal subspaces. As a result, CS could jointly optimize data sampling rates, onboard storage requirements, and communication data payloads.

In Operational Modal Analysis (OMA) applications [5], the integrity assessment task consists in the continuous monitoring of frequency-based quantities referred to as modal parameters (i.e. natural frequencies and mode shapes), as schematically sketched in Fig. 1. In such cases, the benefit brought about by the adoption of CS techniques is twofold. On one side, compression allows to reduce the bandwidth requirements imposed by voluminous raw SHM data on the network, without impinging on the quality of the maintenance process itself [6]. On the other hand, data analytics can also benefit from new processing methods, which are capable to extract meaningful 
features directly from compressed measurements, hence minimizing the possible latency implied by the processing of big data [7].

CS strategies can be fruitfully employed both for wired and wireless architectures. In the former case, in particular for multiplex wiring systems, the reduction of data packets to be transmitted paves the way to an increase in the number of sensors simultaneously connected to the same central unit. In wireless communication systems, a CS approach is beneficial not only to support the design of energy aware solutions by extending battery life, but also to inherently tackle the problem of data loss due to transmission fails [8]. However, the main focus of this work is to exploit CS strategies as a means to optimize the encoding mechanism.

\section{A. Related works}

In the last few years, many researchers investigated the suitability of different CS techniques in the vibration-based SHM context. Among the most representative examples, authors in [9] explored the advantages of a combined spectrotemporal compression approach (STCS) leveraging a bidirectional feature transmission scheme. A distributed CS paradigm was proposed, which involves several leaf nodes under the orchestration of a master aggregating unit. Alongside, it has recently been demonstrated that the data recovery problem can be formalized into a standard supervised-learning task [10], therefore featuring a tight interweaving between standard CS operations and subspace learning techniques driven by artificial intelligence [11]. Alternatively, a CS-based sparse coding strategy was efficiently complemented with a nonconvex shrinkage algorithm to reconstruct the original data from incomplete measurements in the field of large-span structures [12]. Furthermore, Yang \& Nagarajaiah explored the possibility to extract mode shapes directly from low-rate random measurements by tackling the modal identification task with a CS-driven Blind Source Separation (BSS-CS) approach [13].

All the mentioned CS solutions exploit standard compression matrix, whose entries can either be taken from common Gaussian or Bernoulli distributions [14], or obey to random sampling (RND-CS), namely the selection of an arbitrarilyindexed subset of samples [15]. RND-CS-based sensing strategies are inherently suitable to counteract the additional loss of packets affecting the communication channel. Indeed, assuming that data loss can be modeled as a random stochastic process, its effect can be seen as the output of a random sampler that arbitrarily selects few packets from the whole payload.

Alternative solutions for data compression in OMA scenarios are the power spectrum blind sampling (PSBS) and the random demodulator (RD) approach, which are sub-Nyquist techniques specifically suited for hardware implementations. One noticeable example is the method proposed in [16], which extracts second order statistics (i.e. modal information) directly from multi-coset sampled data without any additional hypothesis on the nature of the involved phenomenon. Conversely, in [17], random alternation of samples' sign is combined with low-pass filtering and low-rate sampling. Such technique was proposed to account for data loss, and it is based on accumulate and dump operations that can be readily implemented in smart sensor platforms.

The compression of vibration signals can also be tackled as a pure eigenvalue problem by applying the Principal Component Analysis (PCA) [7]. Eigenvalue strategies compress data by projecting them onto the signal subspace spanned by the directions related to the most energetic components. For example, authors in [18] stressed the potential of PCA for feature compression and reconstruction in the context of predictive maintenance and anomaly detection. These methods show good recovery performances even under significant compression ratios. However, their main limitation is in the huge amount of data they require for the statistical characterization of the input signal energy profile, which implies exhaustive and accurate baseline measurement campaigns necessary to characterize the structure in pristine conditions [19].

\section{B. Contribution}

In this work, a rakeness-based CS (Rak-CS) [20] approach is considered, for its unique capability to adapt the statistical distribution of the sensing mechanism in a way that maximizes the total energy which may be "raked" from the different components of the input signal. To the best of the authors' knowledge, this work represents the first attempt to map the Rak-CS technique in the context of OMA-based vibration diagnostics. The proposed method is inspired by the close analogy between the sparse-and-localized signal assumption which characterizes the Rak-CS paradigm and the energy distribution of vibration data in the frequency domain, which is concentrated around a few and highly localized modal peaks. A distinctive advantage of Rak-CS consists of its relatively soft exploration-localization trade-off [21], which prevents the compression scheme to overspecialize data against potential spectral variations, which are very likely to occur in real scenarios.

Moreover, a novel model-assisted approach (MRak-CS) is employed, in which numerical estimation of the structural dynamics are exploited to design the compressive sensing mechanism. This solution prevents the necessity to collect large training datasets for the same task. In this sense, and in line with the so-called theory-guided data science paradigm [22], the knowledge derived by numerical simulations or by semi-analytical methods is seamlessly blended with the signal statistics characterization process. Finally, the design of an application-specific representation basis to favor the sparsification of vibration data is here presented.

The paper is organized as follows. In Section II the theoretical background behind Rak-CS data compression as well as its model-assisted version are detailed. Thereafter, the main principles of a novel algorithm targeted on the design of a sparse representation domain are introduced. The architectural characteristics of a low-power and miniaturized monitoring network built on an inertial measurement platform are described in Section III. hence discussing how the data compression and reconstruction tasks could be efficiently 
distributed obeying to an "edge computing" paradigm. Section IV pertains to the experimental validation of the proposed monitoring architecture, whilst results are discussed in Section $\mathrm{V}$. Conclusions are finally drawn at the end.

\section{BASICS OF CS}

The enabling assumption behind the applicability of CS strategies is the sparsity-compressibility condition of the underlying signal in a specific domain [4]. In mathematical form, the previous hypothesis states that a basis $\Psi \in \mathbb{R}^{n \times n}$ exists in which a signal instance $x \in \mathbb{R}^{n}$ can be accurately approximated by at most $k \ll n$ non-negligible components belonging to the signal support, i.e. $x=\Psi c$ ( $c$ being the $n$-dimensional coefficient vector). Hence, by harnessing this signal prior, the information hidden within raw acquired data can be condensed into a more representative measurement vector $y \in \mathbb{R}^{m}$ deriving from the linear combination of $m \ll n$ samples by means of a sensing matrix $S \in \mathbb{R}^{m \times n}$, namely $y=S \Psi c$.

The recovery stage can be accomplished by solving a convex optimization problem, such as the Basis Pursuit Denoising (BPDN) problem [14], the solution of which consists of the set of sparsest coefficient vector $\hat{c}$ guaranteeing the highest accuracy between the sensed and the currently predicted measurement vector $\hat{y}=S \Psi \hat{c}$. The original signal is finally reconstructed as $\hat{x}=\Psi \hat{c}$. Convex or gradient-based solvers are available to this purpose, such as the SPGL1 11 algorithm employed in this work.

\section{A. Rak-CS: a model-assisted approach}

With respect to standard CS theory [14], that adopts a measurement matrix $S$ whose entries are instances of independent zero-mean Gaussian random variables, the Rak-CS approach [21] generates each row $s$ of the measurement matrix $S$ as a zero-mean random vector, but with a correlation profile $C^{s}=\mathbb{E}\left[s s^{T}\right]$ tightly matched to the second-order statistic $C^{x}=\mathbb{E}\left[x x^{T}\right]$ of the raw input vector $x$. The method relies on the maximization of the rakeness quantity

$$
\rho(s, x)=\operatorname{tr}\left(C^{s} C^{x}\right)
$$

which measures the average energy of the projections of instances of $x$ over independently drawn rows of $S(\operatorname{tr}(\cdot)$ stands for the matrix trace operator) [20]. The analytical solution of the Rak-CS problem discussed in [20] yields the correlation profile of the sensing matrix to coincide with

$$
C^{s}=\frac{n}{2}\left(\frac{C^{x}}{\operatorname{tr}\left(C^{x}\right)}+\frac{I_{n}}{n}\right)
$$

where $I_{n}$ indicates an $n \times n$ identity matrix.

In the rakeness approach, a fundamental step is the computation of the correlation matrix $C^{x}$ of the vectors to be acquired. This matrix is typically estimated from a sufficiently large number of signal instances which are expected to be representative of the different measured realizations [21]. Alternatively, for MRak-CS, $C^{x}$ is synthetically derived by

${ }^{1}$ https://www.cs.ubc.ca/ mpf/spgl1/index.html

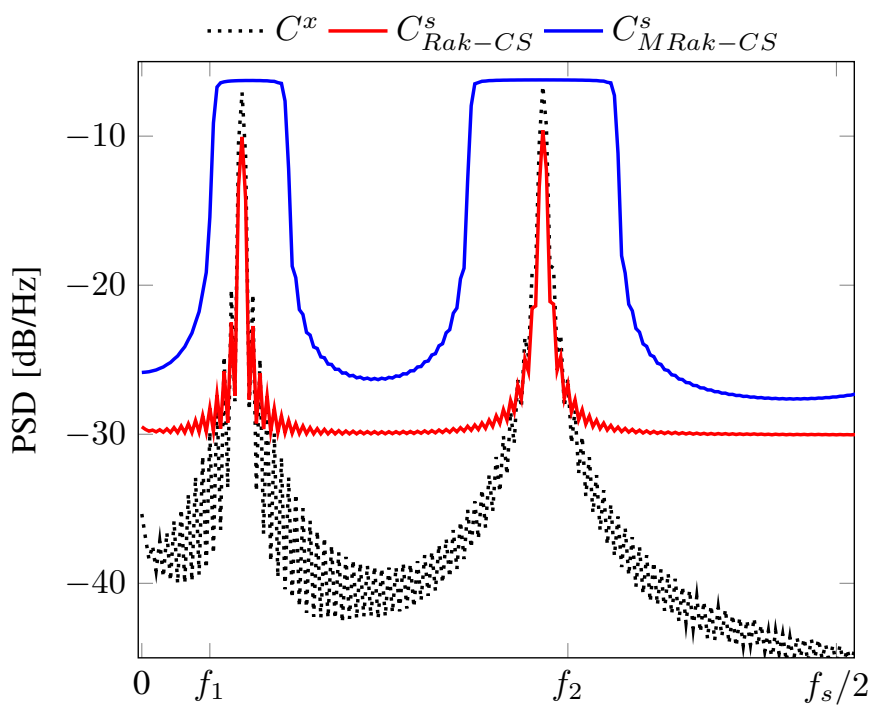

Fig. 2. Spectral profiles of the Rak-CS (red) and MRak-CS (blue) compression matrices superimposed to the energy distribution of the input signal (black curve).

hinging on a priori considerations. If an approximate model of the dynamic response of the structure is available, a condition that usually holds for the majority of industrial and civil plants, a simplified correlation profile can be analytically designed, as detailed in the following.

Let's assume $P$ modes are expected to identify the spectral signature of the structure. Coherently, $P$ rectangular frequency bins are designed, whose spectral width $W_{p}$ is related to the modal frequency $f_{p}$ so that the quality factor $Q=f_{p} / W_{p}$ is constant. Then, since $C^{x}$ should approximately be a positive semi-definite Toeplitz matrix, the value on its $i$-th diagonal is computed as

$$
C^{x}[i]=\sum_{p=1}^{P} \int_{f_{p}-\tau W_{p}}^{f_{p}+(1-\tau) W_{p}} \cos (2 \pi f i) d f
$$

where $\tau$ is a parameter which can be used to offset the central frequency of the bin w.r.t. the modal frequency. Such parameter is introduced to adapt the sensing matrix to the typical evolution of the spectral content in vibration signals. Indeed, in many practical scenarios, the modal frequencies tend to downshift by effect of the stiffness reduction induced by damages. In these cases, it is suggested to select this parameter so that $0.5<\tau<1$. Conversely, when it is impossible to predict how the modal frequencies will evolve, the best option is to select $\tau=0.5$. Under this circumstance, the central frequency of the bin will be coincident with $f_{p}$.

Once the input matrix correlation profile is defined, the eigenvalues and eigenvectors of $C^{s}$ are computed as illustrated in [20]. In Fig. 2, the Power Spectral Density (PSD) of the Rak-CS (red line) and MRak-CS (blue line) compression matrices for a simple problem are enclosed, to show how the two design procedures may adapt to the second-order statistics of the input signal (black dotted curve).

The described strategy can be extended to the case of multiple, closely spaced modal frequencies. If this condition 
applies, the input signal correlation matrix in Eq. (3) can be constructed by selecting $f_{p}$ as the central frequency of the band $B_{p}$ in which the modal frequencies are expected to be located, while $\tau$ and $W_{p}$ are selected so that the spectrum of the sensing matrix is flat in $B_{p}$.

The pros and cons of the data compression methods discussed in this paper w.r.t. the MRak-CS strategy are summarized in Table II. In essence, MRak-CS is a hybrid approach between the purely random sampling which characterizes the conventional CS and the data compression based on an extensive preliminary characterization of the structural response, as in PCA methods. There is a trade-off involved in the MRak-CS implementation, that is related to the definition of the frequency bin span: the larger the span, the closer the compression performance will be to the (relatively poor) one achieved with standard CS strategies. Conversely, by narrowing the span, the attainable compression level can be improved, but the risk is to loose the capability to track the changes in the structural dynamics. Noteworthy, the same risk characterizes PCA-based compression procedures.

Nonetheless, as it will be shown in the results section, the MRak-CS approach has great potentialities for SHM implementations thanks to its adaptability, at least when the numerical modeling of the structure can be implemented with sufficient accuracy.

\section{B. Structurally-shaped sparsity basis}

The selection of the optimal sparsity basis plays a crucial role in boosting compression performance as much as possible. Vibration signals with low damping factors are sparse in the Fourier domain [24]. For these reasons, the Discrete Cosine Transform (DCT) or the Discrete Fourier Transform (DFT) matrices have been conventionally chosen as sparsifying bases [10], [25]. Alternatively, Wavelet bases have been proposed [26] to better track non-stationary phenomena.

More recently, several approaches were explored to specifically design a best-adapted signal domain representation, including sparse coding [27] and dictionary learning [28]. The Wavelet Packet Transform (WPT) is particularly suited to perform this adaptation to the signal characteristics in a very flexible way [29]. The WPT is conceived as a nested filtering operator applied to both the detail (i.e. high-frequency) and approximation (i.e. low-frequency) components. The sequence (tree) of filtering stages can be purposely pruned to match the intrinsic multi-scale nature of the signal; in these terms, the WPT operator can provide an efficient representation for signals specifically localized at distinct sub-bands distributed over the entire spectrum. Thereby, it can be effectively combined with the Rakeness-based CS method which leverages the signal localization property for the sensing matrix design. Such optimization can be performed by learning the most suited representation from uncompressed [30] or compressed data [31]. Even if these methods proved to be robust and promising for an effective signal recovery process, they require a consistent amount of data to be used during the training phase.

In this work, we took advantage of structural numerical models to shape the WPT decomposition process according

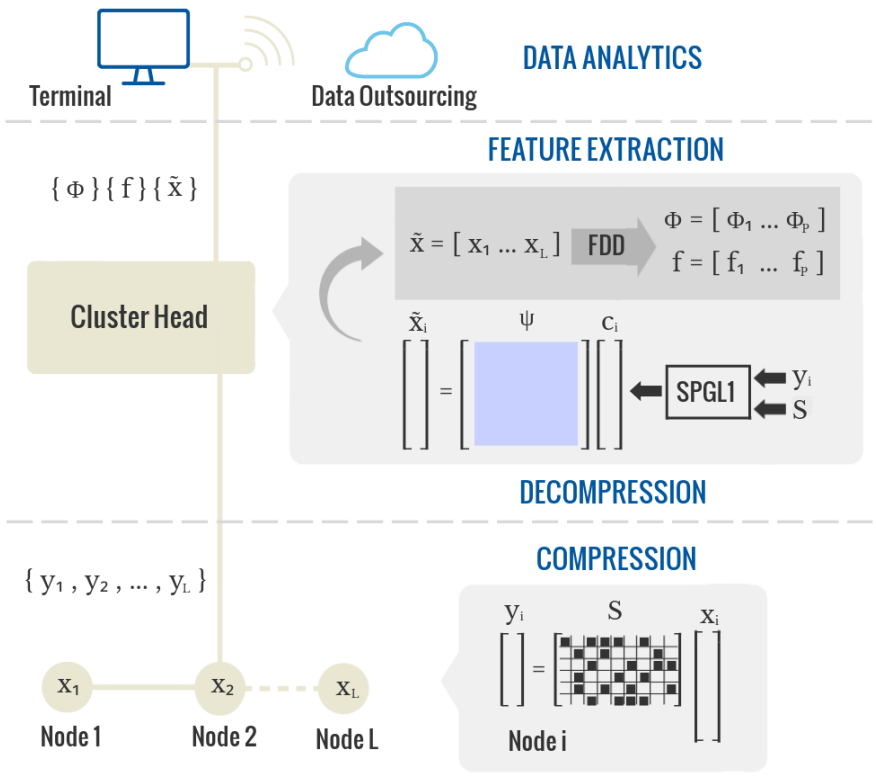

Fig. 3. System architecture for vibration-based SHM. In light-gray background, a schematic representation of the signal processing procedures necessary for the compression and decompression stages; conversely, the dark-gray box refers to the pure OMA feature extraction process.

with the PSD of vibration signals. The WPT adaptation procedure is organized around two successive phases, and returns the decomposition level and the frequency interval for each wavelet packet:

1) select the decomposition level $j$ as the maximum value allowing one wavelet packet to capture the first modal frequency while keeping the corresponding frequency bin $\Delta_{f}=F s / 2^{j+1}$ below the distance $\Delta_{f}^{\min }$ between the closest modal frequencies. The minimum span of the WPT frequency bin has an inferior bound given by the length of the frame size $(n): \Delta_{f}^{\min }=\frac{F_{s}}{2^{\log _{2} n+1}}$. In turn, $n$ is limited by the memory space available in embedded sensors; thus, attainable values for $\Delta_{f}^{\min }$ range from one to few tenths of Hertz, depending on the adopted sampling rate. In typical OMA applications, such values are usually sufficient to locate strongly coupled modal frequencies in separated WPT bins. The overcomplete wavelet tree is then created up to depth $j$, yielding to $2^{j}$ successive wavelet packets, each of them being identified by a corresponding frequency interval and width. Define with $B^{*}$ the ensemble of packet bands falling outside the frequency region of interests $W=\bigcup_{p=1}^{P} B_{p}=\bigcup_{p=1}^{P}\left[f_{p}-\tau W_{p}, f_{p}+(1-\tau) W_{p}\right]$, i.e.
$B^{*} \cap W=\emptyset$.

2) climb back the full tree level-by-level. At each filtering stage, merge those children packets associated with a father node whose frequency band belongs to $B^{*}$. Repeat this procedure until the dyadic grouping is permitted. 
TABLE I

COMPARISON BETWEEN DIFFERENT METHODS FOR OMA-BASED DATA COMPRESSION: $\boldsymbol{V}$ AND $\boldsymbol{X}$ INDICATE WHETHER THE CORRESPONDING STRATEGY IS IMPLEMENTED IN AN UNSUPERVISED OR SUPERVISED MANNER, RESPECTIVELY.

\begin{tabular}{|c|c|c|c|c|}
\hline Method & Advantages & Drawbacks & Type & Ref. \\
\hline STCS & $\begin{array}{l}\text { - Well-balanced resource distribution } \\
\text { - Sparsity adapted at acquisition time }\end{array}$ & $\begin{array}{l}\text { - Additional payload due to the transmission of coor- } \\
\text { dination variables } \\
\text { - High computational complexity of the data recovery } \\
\text { algorithm }\end{array}$ & $\checkmark$ & 9 \\
\hline RND-CS & $\begin{array}{l}\text { - Straightforward implementation of the decoder } \\
\text { - Suitable for data loss recovery }\end{array}$ & - High sensitivity to input noise level & $\checkmark$ & 23 \\
\hline BSS-CS & - Mode shapes recovered from low-rate measurements & $\begin{array}{l}\text { - High computational complexity } \\
\text { - Number of observable modes inferior to the cluster } \\
\text { size }\end{array}$ & $\checkmark$ & 13 \\
\hline PSBS & $\begin{array}{l}\text { - Modal parameters extracted directly from com- } \\
\text { pressed data } \\
\text { - Low computational complexity of the encoder }\end{array}$ & $\begin{array}{l}\text { - High computational complexity and memory require- } \\
\text { ments for the decoder }\end{array}$ & $\checkmark$ & 16 \\
\hline $\mathrm{RD}$ & $\begin{array}{l}\text { - Low computational cost of the encoder } \\
\text { - Low memory requirements } \\
\text { - Data loss inherently handled }\end{array}$ & $\begin{array}{l}\text { - Poor performance in the recovery of noisy acquisi- } \\
\text { tions } \\
\text { - Short frame length is required }\end{array}$ & $\checkmark$ & 17 \\
\hline MRak-CS & $\begin{array}{l}\text { - Soft adaptation process to the input signal energy } \\
\text { profile } \\
\text { - Robustness with respect to structural variations } \\
\text { - Low computational cost for the encoder }\end{array}$ & $\begin{array}{l}\text { - Structural models are required to design the sensing } \\
\text { matrix }\end{array}$ & $x$ & $\begin{array}{l}\text { This } \\
\text { work }\end{array}$ \\
\hline
\end{tabular}

\section{SHM ARCHITECTURE}

A low-power and low-cost Smart Sensor Network (SSN) [32] was used to experimentally validate the proposed approach. Each peripheral sensor node is equipped with a microcontroller unit (MCU) and a tri-axial Micro-electromechanical System (MEMS) inertial measurement unit. Peripheral Sensor Nodes (leaves) are connected to a Cluster Head $(\mathrm{CH})$ node in charge of data management and feature extraction.

The data storage capabilities of the leaf nodes are limited to $40 \mathrm{KiB}$ SRAM and $256 \mathrm{KiB}$ FLASH memory so it is fundamental to develop effective compression compatible with the available computational resources. The framework depicted in Fig. 3 features a balanced distribution of the embedded resources of the network for streaming data compression. Each leaf node $\left(\mathrm{Node}_{i}\right)$ independently performs the data compression procedure by operating over successive segments, each comprising $n$ samples of the acquired signal. The sensing matrix $S$, which is populated with digitized Rak-derived antipodal entries (i.e. $S_{i, j} \in\{-1,1\}$ ) [21] to ease the entailed vector-matrix operations, is stored in the MCU node memory during the network start-up phase. Thanks to the relatively loose Rak-based adaptation procedures, it is not necessary to update $S$ even in case of strong environmental variations.

The signal recovery and modal feature estimation stages are performed by the $\mathrm{CH}$, which first recovers node-related data in a sequential way and then processes them in a centralized manner to extract the sought modal features. The estimated parameters can be finally forwarded to a remotely connected station or stored in a cloud database for further analysis.

\section{EXPERIMENTAL VALIDATION}

\section{A. Materials}

A pinned-pinned steel beam was instrumented with a chain of six nodes mastered by a $\mathrm{CH}$, as schematically depicted in Fig. 4. The beam is characterized by the following mechanical and geometrical properties: material density $\rho=7880 \mathrm{~kg} / \mathrm{m}^{3}$, Young's modulus $E=200 \mathrm{GPa}$, beam height $h=10 \mathrm{~mm}$. The three fundamental modes of such structure are localised at the following frequencies: $f_{1}=5.52 \mathrm{~Hz}, f_{2}=22.08 \mathrm{~Hz}$ and $f_{3}=49.68 \mathrm{~Hz}$. These values can be predicted either by a Finite Element Model (FEM) analysis or by the following closed analytic formula [33]:

$$
f_{p}=\left(\frac{p}{L}\right)^{2} \frac{\pi h}{4} \sqrt{\frac{E}{3 \rho}}
$$

Frequency components above $100 \mathrm{~Hz}$ were neglected in the following structural integrity characterization.

\section{B. Methods}

Each time series was acquired at a data rate of $F_{s}=200 \mathrm{~Hz}$ over a sampling period of 75 s (i.e. 15000 samples in each iteration). The beam was left to oscillate under pure environmental noise (i.e. ground motion excitation), therefore mimicking the classical exciting mechanism required by output-only modal analysis. Moreover, since the bounding conditions force the structure to vibrate along the vertical direction, devices were programmed to collect accelerations only on the $z$ axis.

The frame size was fixed to $n=512$ samples, a quantity that corresponds to the best compromise between the necessary 


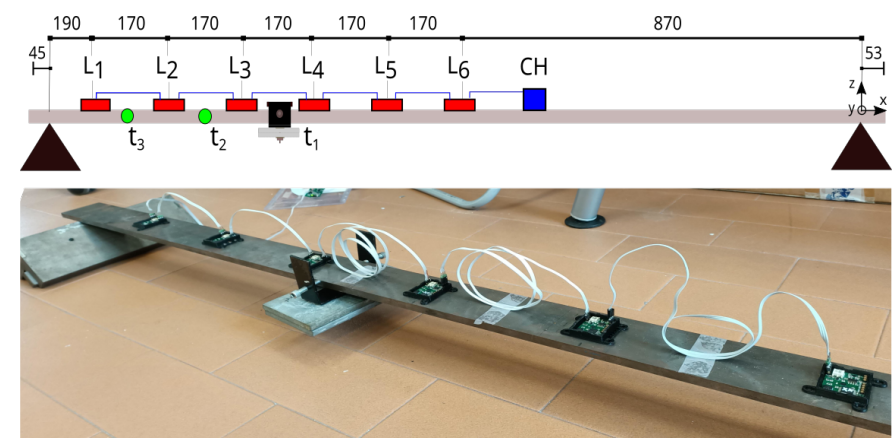

Fig. 4. Experimental setup: sensor installation plan (all dimensions in millimeters) inclusive of the damaging mechanism and positions.

spectral resolution and the sensor node memory capability. The number of measurements $m$ sets the compression ratio $C R=n / m$. In particular, the results achieved by varying from $C R=4$ to $C R=10$ are reported.

Two datasets were acquired in nominal and altered structural configurations. The former, associated to the healthy status, was used to estimate off-line the correlation matrix corresponding to the given structure, as required by the RakCS sampling mechanism. Conversely, the latter was employed during the real testing phase. For this reason, acceleration data deriving from intact vibration behavior were collected along with those pertaining to structural anomalies, which were induced by laterally hinging a progressively heavier dead mass ( $\left.m_{A}=1.042 \mathrm{~kg}, m_{B}=1.808 \mathrm{~kg}\right)$ to the beam at different positions $\left(t_{1}=1.8 \mathrm{~m}, t_{2}=1.6 \mathrm{~m}, t_{3}=1.4 \mathrm{~m}\right)$. Thereafter, the caption $m_{d} @ t_{g}$ will be used to indicate a generic damage configuration resulting from mass $m_{d}$ hinged at position $t_{g}$, with $d \in\{A, B\}$ and $g \in\{1,2,3\}$.

As prescribed by the theory of Structural Dynamics for the class of beam-like structures, vibration frequencies tend to decrease in response to mass and stiffness variations induced by aging [34]. For this reason, considering the MRak-CS approach, the parameter $\tau$ was assumed equal to 0.75 to better track possible frequency downshifts. Accordingly, a band ratio $Q=5$ was chosen to ensure a significant frequency inspection in the neighborhood of the estimated peaks.

For the pure modal estimation task, the well-established Frequency Domain Decomposition ${ }^{2}$ (FDD) technique [5] was adopted to extract the set of natural frequencies $f_{p}$ and the associated mode shapes $\Phi_{p}$. The matching between the theoretical modal frequencies predicted by Eq. 4 and those extracted with FDD was assessed with experimental data coming from compression-free nominal tests. The extracted frequencies were $\hat{f}_{1}=5.47 \mathrm{~Hz}, \hat{f}_{2}=22.07 \mathrm{~Hz}$ and $\hat{f}_{3}=$ $49.37 \mathrm{~Hz}$, showing a maximum relative percentage error w.r.t the theoretical values equal to $\varepsilon_{1}=100\left(1-\frac{\hat{f}_{1}}{f_{1}}\right)=0.843 \%$ in correspondence of the first mode.

\footnotetext{
${ }^{2}$ This procedure also returns the sign of the inspected mode shapes. For this reason, it was chosen over the pure modal peak interpolation presented in Fig. 1 ]
}

\section{Results}

Three main objectives were pursued within the validation phase: $(i)$ proving the superiority of the devised WPT sparsifying basis w.r.t. the classical DCT operator, (ii) assessing the validity of both the Rak-CS and MRak-CS scheme in the framework of vibration-based structural inspection, and (iii) comparing the performance of the proposed sampling schemes against basic eigenvalue-based methodologies and alternative CS-based solutions for OMA, in terms of compression ratio and quality of the reconstructed structural information.

To this purpose, the classical PCA [19] was considered as the main competitor among the standard eigenvalue approaches because of its fully-symmetric and low-complexity approach. Indeed, both the PCA encoding and decoding side can be easily implemented through a simple matrix-vector multiplication, a procedural characteristic which corroborated its success for simple deployment on-sensor. In this case, the compression factor $C R$ should be intended as the ratio between the total frame size and the number of preserved principal components.

Among alternative CS implementations, the approach proposed in [23] can be considered as a benchmark method since it represents the first example of effective long-term monitoring with CS-enabled sensor nodes. This strategy does not rely on any structural priors and it is based on random sampling: $m$ out of $n$ randomly sampled rows of the Inverse DFT matrix are used to assemble the sensing matrix. In this work, RND-CS is implemented by using the WPT as sparsity basis and the SPGL1 as data recovery algorithm, i.e. the same configuration adopted for the Rakness-based frameworks.

\section{A. Sparsity basis: DCT vs WPT}

Exploiting the structural priors detailed in Sec. IV-A as inputs for the adapted WPT decomposition process discussed in Sec. II-B the sparsity basis $\Psi$ was derived. In particular, the Fejerr-Korovkin mother wavelet with 22 taps was chosen because of its highly vanishing high-order lobes [35] (i.e. minimal filter overlap between adjacent packets) with respect to smoother alternatives (e.g. Daubechies, Symlet). The decomposition level was set equal to 6 , consequently the minimum packet bandwidth is $200 / 2^{7}=1.57 \mathrm{~Hz}$ which allows a good discrimination of the modal components.

To quantify the sparsifying effect of the signal representation domain [36], the Gini index for an $n$-dimensional vector $a$ was selected:

$$
G=1-2 \sum_{k=1}^{n} \frac{a_{k}}{\|a\|_{1}}\left(\frac{n-k+0.5}{n}\right)
$$

The Gini index was preferred over energy-dependent alternatives because of its higher statistical relevance in presence of noisy measurements $\left(\|\cdot\|_{p}\right.$ stands for the $\ell_{p}$ vector norm). This is the case of the considered scenario, where recorded acceleration data are characterized by weak amplitudes as a direct consequence of the considered beam loading conditions.

In this paper, vector $a$ consists in the projection coefficients of the acceleration data calculated as $a=\Psi^{-1} x$. In particular, 


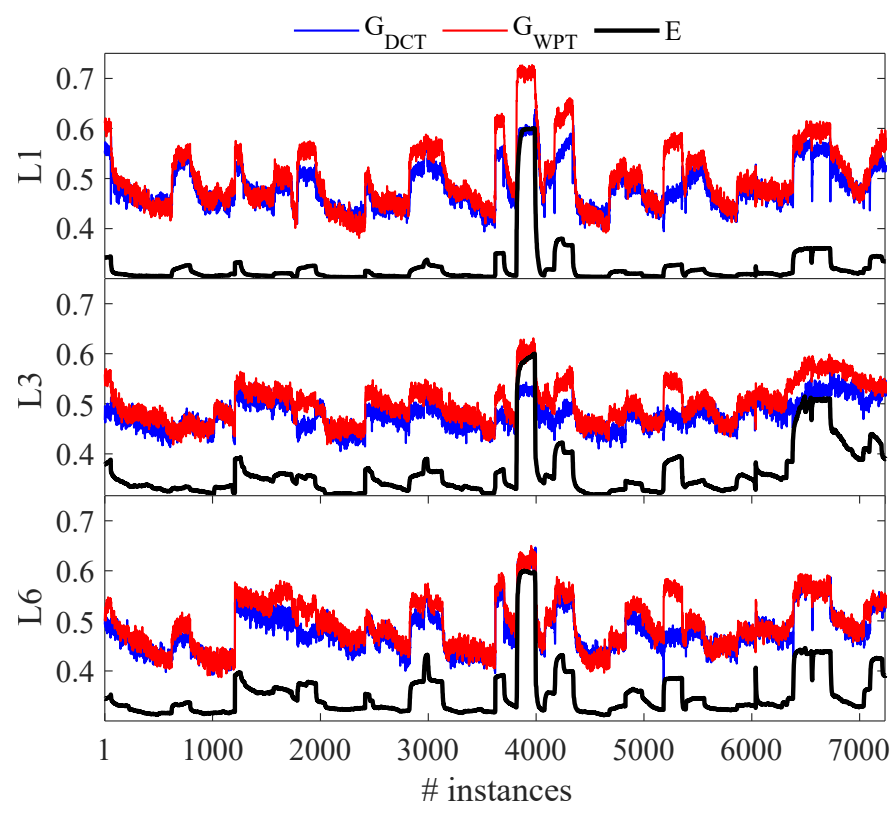

Fig. 5. Trends in Gini index for WPT (red) and DCT (blue) transformed acceleration coefficients over different signal instances, superimposed to the normalised and rescaled signal energy (black curve) in the original time domain. Results are presented for sensing positions $L_{1}, L_{2}, L_{3}$.

the Gini term was computed both for the DCT and WPT representations for multiple time frames and sampling locations. Such a multi-sensor assessment procedure was necessary to prevent the analysis from being influenced by sensing positions proximal to the nodal points of the investigated mode shapes, in which the energy of the modal components is vanishing.

The outcomes of the sparsity analysis are depicted in Fig. 5 in which each of the three panels represents the actual trends in the $G$ index for the adapted WPT (red line $-G_{W P T}$ ) and DCT (blue line - $G_{D C T}$ ) basis over subsequent signal frames for sensor locations $L_{1}, L_{3}, L_{6}$. As $G$ approximates the unit value, the sparsity ratio is more pronounced. The global frame energy $E_{g}$ (black curve) is also illustrated, so that the obtained sparsity indicators can be compared to the magnitude of the measured acceleration data in the original time domain 3 Apart from low energy segments, in which the blue and red curves are almost overlapped, the sparsifying effect of the WPT basis is clearly superior for all the considered sensor positions, with $G_{W P T}$ larger than $G_{D C T}$ of more than 0.1 points on average.

\section{B. Compression approach: Rakness-based vs benchmark so- lutions}

The Modal Assurance Criterion (MAC) metric [5], which measures the level of superimposition between two mode shape vectors, was selected to assess the quality of the signal recovery as a function of the compression ratio. When the MAC value is equal to 0.90 or larger (MAC $=1$ corresponds to perfect fitting), the recovery is deemed to be sufficiently accurate [34].

\footnotetext{
${ }^{3}$ For pure visualization purposes, this parameter has been rescaled to be comparable with Gini index values.
}

The performance was evaluated in all the tested configurations (nominal and altered). Exemplary spectra in nominal conditions for one data record are reported in the upper charts of Fig. 6, in which each of the three charts relates, from left to the right, to the Rak-CS, MRak-CS and PCA reconstructed acceleration data. The curves in each panel indicate the frequency distribution of the Cross-Power Spectral Density (CPSD) matrix among the six sampling positions, stepping from lower (blue) to higher (red) CR values, overlapped to the raw measurements' profile (black).

One fundamental result needs to be underlined, which is the filtering effect inherent to the PCA strategy. Noticeably, the structural information which is retained after the PCA compression/decompression process is limited to the acceleration content appearing in the estimated input correlation profile during the training phase. Consequently, spurious components, which can be induced either by frequency shifts from pristine vibration conditions or by outbreaks of unexpected modal components due to possible structural deterioration, are canceled out by the signal compression/decompression. Conversely, the Rak-CS and MRak-CS can capture these unpredictable but highly probable vibration anomalies.

It is worth noting that the input signal correlation matrix used for altered conditions is the same that was adopted for the nominal ones. In this way, it was possible to evaluate how the distinct encoding/ decoding mechanisms may cope with spectral changes which may occur in long-term monitoring tasks. For instance, the third vibration mode appearing in the panels altered with a mass $m_{B}$ hinged at position $t_{2}$ (highlighted in the bottom subplots of Fig. 6 with yellow diamond markers) is downshifted from $49.68 \mathrm{~Hz}$ in nominal conditions to $44.39 \mathrm{~Hz}$, corresponding to a frequency reduction above $10 \%$. Notably, such a significant change cannot be tracked by the PCA strategy, while Rak-CS and MRak-CS are capable to detect such mode even for high CRs.

MAC factors computed by correlating mode shapes coming from uncompressed vs recovered measurements for the three dominant vibration modes are reported in Table III As can be seen, a poor reconstruction performance characterizes the RND-CS method: very low MAC values (often below 0.9) are achieved in all the considered structural configurations if CR is higher than 5. This is coherent with the compression levels reported in previous studies conducted on long-span structures [23].

The performance of PCA compression strategy is substantially different. In this case, as long as variations in the frequency peak localizations are modest w.r.t. pristine status and effectively represented by the eigenvalues belonging to the subset of preserved components, PCA outperforms the Rak ness-based alternatives. Indeed, in these cases, MAC factor is equal to one even for the highest compression ratios. On the other hand, PCA performance degrades when the structural response varies due to strong perturbations. For example, MAC values related to PCA for the third and less energetic mode are smaller than 0.9 for all the considered CRs and the decoded information loses any consistency with respect to the original signal. Under the same circumstances, the Rakeness-based methods are capable to accurately reconstruct 


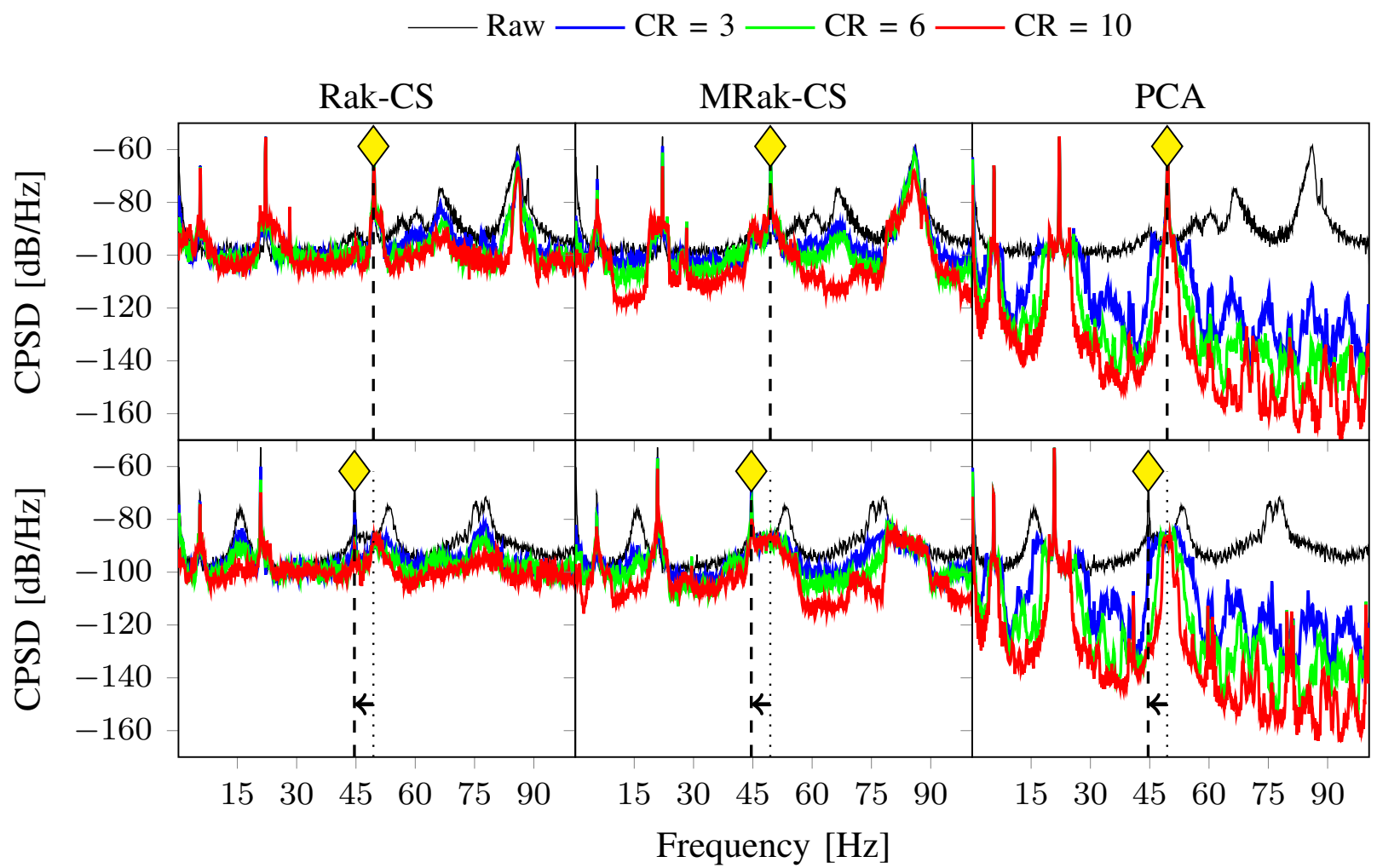

Fig. 6. Correlation profile of the CPSD matrix for the considered compression methodologies as a function of increasing CR values in nominal ( top line) and altered ( bottom line) conditions. Yellow markers are also included in order to better track identify the frequency shift experienced by the third vibration mode.

the third mode. An important consideration is related to the fact that high-frequency modes are typically more significant for the characterization of the structural integrity due to their better sensitivity to structural damages. Therefore, the correct reconstruction of their associated mode shapes is of the utmost importance.

In the comparison among the Rak-driven solutions, it is possible to observe a more pronounced denoising effect of the MRak-Cs method, while the noise floor of Rak-CS is slightly higher. This effect is more evident for higher CRs. The improvement brought by the adoption of the MRak-CS design is also evident in the results reported in Table [II In detail, MAC values achieved with MRak-CS are larger than those attained with Rak-CS in almost all the altered cases. In particular, it might be shown that MRak-CS provides sufficient MAC factors (i.e. MAC $\geq 0.9$ ) when $\mathrm{CR}$ is increased up to $C R=7$, which guarantees a sufficient modal consistency for all the inspected modes. This result compares favorably with alternative solutions presented in the literature, where the estimated CR values were consistently below $\mathrm{CR}=5$ [9].

\section{CONCLUSIONS}

In this work, a novel model-based compressive sensing strategy for vibration monitoring was presented. The validity of the proposed data compression system was assessed during an experimental campaign aimed at verifying the integrity conditions of a pinned-pinned steel beam. The obtained results highlight that the concurrent optimization of the signal representation domain together with the advantages of the MRakCS sensing scheme allow for increased compression ratios while attaining superior performances in the quality of the reconstructed structural parameters if compared with conventional approaches. The effectiveness of the method was also shown in defective configurations. Further developments will involve the performance assessment on complex structures, such as long-span bridges and rotating machines, where the reconstruction of full-scale modal parameters is crucial.

\section{REFERENCES}

[1] F. Ubertini, S. Laflamme, E. Chatzi, B. Glisic, and F. Magalhaes, "Dense sensor networks for mesoscale shm: innovations in sensing technologies and signal processing," Measurement Science and Technology, vol. 28, no. 4, p. 040103, 2017.

[2] F. Zonzini, A. Girolami, L. De Marchi, A. Marzani, and D. Brunelli, "Cluster-based vibration analysis of structures with graph signal processing," IEEE Transactions on Industrial Electronics, 2020.

[3] M. Z. A. Bhuiyan, J. Wu, G. Wang, and J. Cao, "Sensing and decision making in cyber-physical systems: The case of structural event monitoring," IEEE Transactions on Industrial Informatics, vol. 12, no. 6, pp. 2103-2114, 2016.

[4] S. Li, L. Da Xu, and X. Wang, "Compressed sensing signal and data acquisition in wireless sensor networks and internet of things," IEEE Transactions on Industrial Informatics, vol. 9, no. 4, pp. 2177-2186, 2012.

[5] C. Rainieri and G. Fabbrocino, "Operational modal analysis of civil engineering structures," Springer, New York, vol. 142, p. 143, 2014.

[6] Z.-X. Hu, Y. Wang, M.-F. Ge, and J. Liu, "Data-driven fault diagnosis method based on compressed sensing and improved multiscale network," IEEE Transactions on Industrial Electronics, vol. 67, no. 4, pp. 3216$3225,2019$. 
TABLE II

MAC VALUES BETWEEN RAW AND RECONSTRUCTED MODAL SHAPES FOR DIFFERENT STRUCTURAL CONFIGURATIONS. FROM TOP TO DOWN GROUP LINES, RND-CS, PCA, RAK-CS AND MRAK-CS RESULTS.

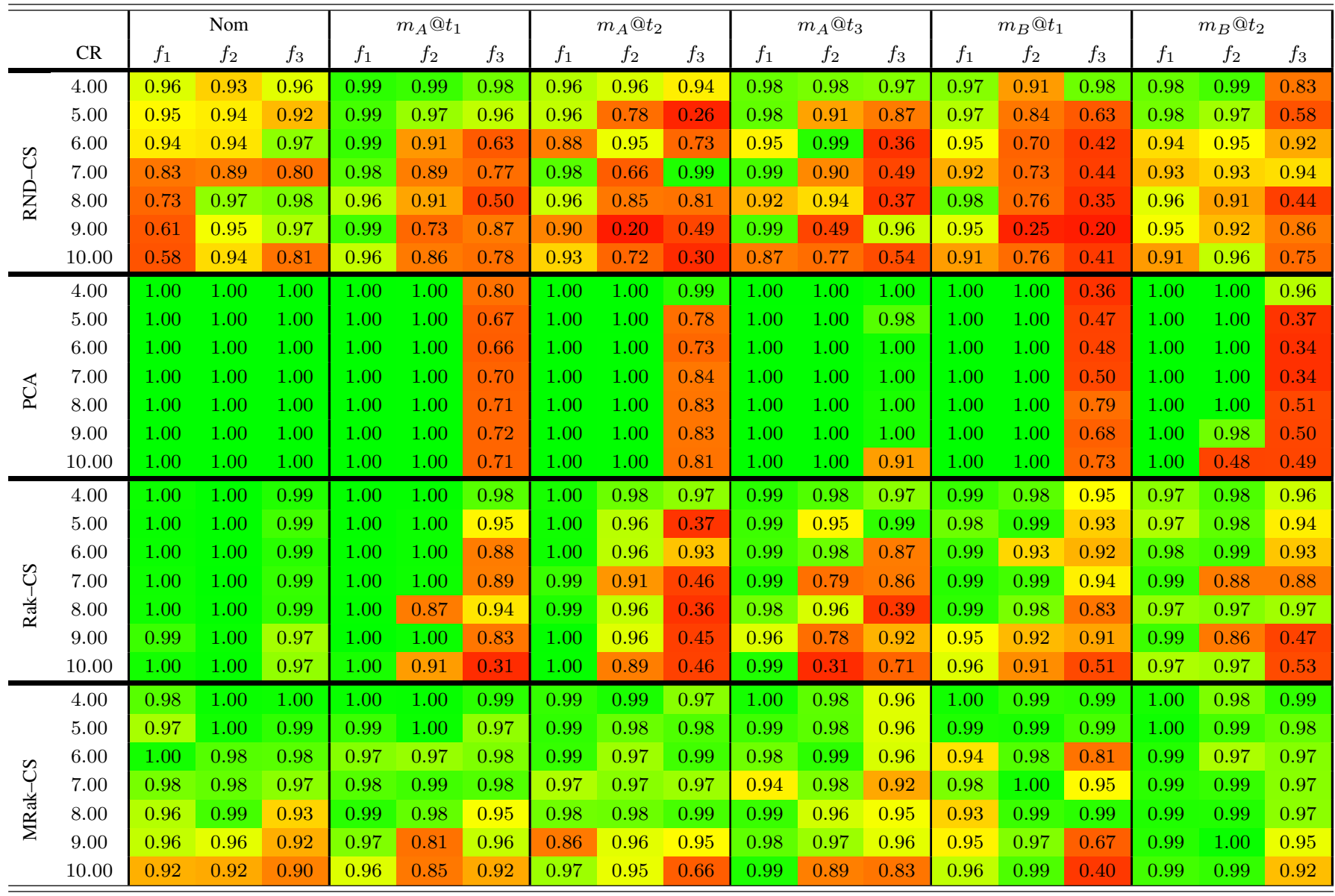

[7] J. Y. Park, M. B. Wakin, and A. C. Gilbert, "Modal analysis with compressive measurements," IEEE Transactions on Signal Processing, vol. 62, no. 7, pp. 1655-1670, 2014.

[8] Y. Bao, H. Li, X. Sun, Y. Yu, and J. Ou, "Compressive sampling-based data loss recovery for wireless sensor networks used in civil structural health monitoring," Structural Health Monitoring, vol. 12, no. 1, pp. 78-95, 2013.

[9] R. Klis and E. N. Chatzi, "Vibration monitoring via spectro-temporal compressive sensing for wireless sensor networks," Structure and Infrastructure Engineering, vol. 13, no. 1, pp. 195-209, 2017.

[10] Y. Bao, Z. Tang, and H. Li, "Compressive-sensing data reconstruction for structural health monitoring: a machine-learning approach," Structural Health Monitoring, vol. 19, no. 1, pp. 293-304, 2020.

[11] H. O. Ahmed and A. K. Nandi, "Three-stage hybrid fault diagnosis for rolling bearings with compressively sampled data and subspace learning techniques," IEEE Transactions on Industrial Electronics, vol. 66, no. 7, pp. 5516-5524, 2018.

[12] J. Yan, H. Peng, Y. Yu, and Y. Luo, "Compressive sensing of wind speed based on non-convex $l_{p}$-norm sparse regularization optimization for structural health monitoring," Engineering Structures, vol. 194, pp. 346-356, 2019.

[13] Y. Yang and S. Nagarajaiah, "Output-only modal identification by compressed sensing: Non-uniform low-rate random sampling," Mechanical Systems and Signal Processing, vol. 56, pp. 15-34, 2015.

[14] E. J. Candes and T. Tao, "Decoding by linear programming," IEEE transactions on information theory, vol. 51, no. 12, pp. 4203-4215, 2005.

[15] S. Salloum, J. Z. Huang, and Y. He, "Random sample partition: a distributed data model for big data analysis," IEEE Transactions on Industrial Informatics, vol. 15, no. 11, pp. 5846-5854, 2019.

[16] K. Gkoktsi and A. Giaralis, "A multi-sensor sub-nyquist power spectrum blind sampling approach for low-power wireless sensors in operational modal analysis applications," Mechanical Systems and Signal Processing, vol. 116, pp. 879-899, 2019.

[17] Z. Zou, Y. Bao, H. Li, B. F. Spencer, and J. Ou, "Embedding compressive sensing-based data loss recovery algorithm into wireless smart sensors for structural health monitoring," IEEE Sensors Journal, vol. 15, no. 2, pp. 797-808, 2014.

[18] A. Marchioni, M. Mangia, F. Pareschi, R. Rovatti, and G. Setti, "Subspace energy monitoring for anomaly detection@ sensor or@ edge," IEEE Internet of Things Journal, 2020.

[19] A. Burrello, A. Marchioni, D. Brunelli, and L. Benini, "Embedding principal component analysis for data reduction in structural health monitoring on low-cost iot gateways," in Proceedings of the 16th ACM International Conference on Computing Frontiers, 2019, pp. 235-239.

[20] M. Mangia, R. Rovatti, and G. Setti, "Rakeness in the design of analog-to-information conversion of sparse and localized signals," IEEE Transactions on Circuits and Systems I: Regular Papers, vol. 59, no. 5, pp. 1001-1014, 2012.

[21] M. Mangia, F. Pareschi, V. Cambareri, R. Rovatti, and G. Setti, Adapted Compressed Sensing for Effective Hardware Implementations: A Design Flow for Signal-Level Optimization of Compressed Sensing Stages. Springer, 2017.

[22] A. Karpatne, G. Atluri, J. H. Faghmous, M. Steinbach, A. Banerjee, A. Ganguly, S. Shekhar, N. Samatova, and V. Kumar, "Theory-guided data science: A new paradigm for scientific discovery from data," IEEE Transactions on Knowledge and Data Engineering, vol. 29, no. 10, pp. 2318-2331, 2017.

[23] S. M. O’Connor, J. P. Lynch, and A. C. Gilbert, "Compressed sensing embedded in an operational wireless sensor network to achieve energy efficiency in long-term monitoring applications," Smart Materials and Structures, vol. 23, no. 8, 2014.

[24] Z. Du, X. Chen, H. Zhang, and B. Yang, "Compressed-sensing-based periodic impulsive feature detection for wind turbine systems," IEEE 
Transactions on Industrial Informatics, vol. 13, no. 6, pp. 2933-2945, 2017.

[25] V. S. G. Thadikemalla and A. S. Gandhi, "A data loss recovery technique using compressive sensing for structural health monitoring applications," KSCE Journal of Civil Engineering, vol. 22, no. 12, pp. 5084-5093, 2018.

[26] Y. Bao, H. Li, X. Sun, Y. Yu, and J. Ou, "Compressive sampling-based data loss recovery for wireless sensor networks used in civil structural health monitoring," Structural Health Monitoring, vol. 12, no. 1, pp. 78-95, 2013.

[27] Y. Li, S. Bu, Z. Liu, and C. Zhang, "Mechanical fault diagnosis of rolling bearing based on locality-constrained sparse coding," in 2015 IEEE Conference on Prognostics and Health Management (PHM). IEEE, 2015, pp. 1-7.

[28] J. Wang, W. Qiao, and L. Qu, "Wind turbine bearing fault diagnosis based on sparse representation of condition monitoring signals," IEEE Transactions on Industry Applications, vol. 55, no. 2, pp. 1844-1852, 2018.

[29] S. Nagarajaiah and B. Basu, "Output only modal identification and structural damage detection using time frequency \& wavelet techniques," Earthquake Engineering and Engineering Vibration, vol. 8, no. 4, pp. 583-605, 2009.

[30] R. R. Coifman and M. V. Wickerhauser, "Entropy-based algorithms for best basis selection," IEEE Transactions on information theory, vol. 38, no. 2, pp. 713-718, 1992

[31] J. M. Duarte-Carvajalino and G. Sapiro, "Learning to sense sparse signals: Simultaneous sensing matrix and sparsifying dictionary optimization," IEEE Transactions on Image Processing, vol. 18, no. 7, pp 1395-1408, 2009.

[32] N. Testoni, C. Aguzzi, V. Arditi, F. Zonzini, L. De Marchi, A. Marzani, and T. S. Cinotti, "A sensor network with embedded data processing and data-to-cloud capabilities for vibration-based real-time shm," Journal of Sensors, 2018.

[33] K. F. Graff, Wave motion in elastic solids. Courier Corporation, 2012.

[34] R. A. Ibrahim, Handbook of Structural Life Assessment. Wiley Online Library, 2017.

[35] M. Nielsen, "On the construction and frequency localization of finite orthogonal quadrature filters," Journal of Approximation Theory, vol. 108 , no. 1, pp. 36-52, 2001.

[36] N. Hurley and S. Rickard, "Comparing measures of sparsity" IEEE Transactions on Information Theory, vol. 55, no. 10, pp. 4723-4741, 2009.

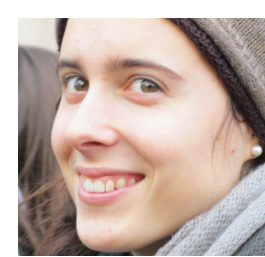

Federica Zonzini (S'19) is currently pursuing the $\mathrm{Ph} . \mathrm{D}$. degree in Engineering and Information Technology for Structural Health and Environmental Monitoring and Risk Management (EIT4SEMM) with the University of Bologna, Italy after receiving her M.Sc. degree in electronic engineering from the same university. Her main research interests include advanced signal processing techniques for structural health monitoring applications, encompassing graph signal processing, data-fusion, compressive sensing and damage assessment.

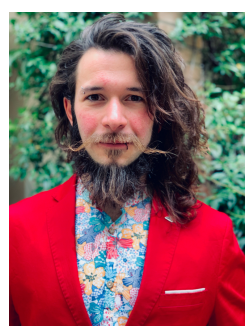

Matteo Zauli (S'20) received the B.S. and the M.S degree in Electronics Engineering at the University of Bologna, Italy. He is currently pursuing the $\mathrm{Ph} . \mathrm{D}$ in Engineering and Information Technology for Structural and Environmental Monitoring and Risk Management (EIT4SEMM) with the University of Bologna. His research interests focus on signal processing, compressed sensing, neural networks, embedded systems development and ultrasonic measurements.

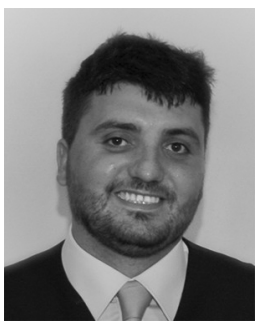

Mauro Mangia (S'09-M'13) received the B.Sc. and M.Sc. degrees in electronic engineering and the Ph.D. degree in information technology from the University of Bologna, Bologna, Italy, in 2005, 2009, and 2013, respectively. He was a Visiting $\mathrm{Ph}$.D. Student with the Ecole Polytechnique Federale de Lausanne in 2009 and 2012. He is currently a Senior Post-Doctoral Researcher with the Statistical Signal Processing Group, University of Bologna. His research interests are in nonlinear systems, machine learning, compressed sensing, anomaly detection, Internet of Things, Big Data analytics and optimization. He was a recipient of the 2013 IEEE CAS Society Guillemin-Cauer Award and of the 2019 IEEE BioCAS Transactions Best Paper Award. He received the Best Student Paper Award at ISCAS2011. He was the Web and Social Media Chair for ISCAS2018.

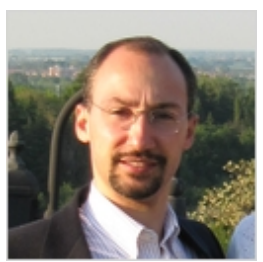

Nicola Testoni received his M.Sc in Microelectronics and his Ph.D in Information Technology from Bologna University in 2004, and 2008 respectively. $\mathrm{He}$ is currently an Adjunct Professor of the Department of Electrical, Electronic, and Information Engineering "Guglielmo Marconi" at Bologna University. His research interests include guided waves, analog circuit design, non-linear signal processing, Wavelet theory and applications, neural signal denoising and event sorting.

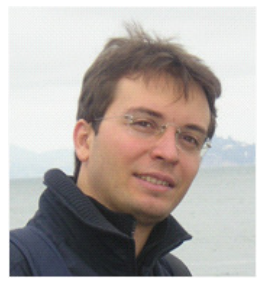

Luca De Marchi (S'10, M'12) is associate professor in Electronics in the Department of Electrical, Electronic, and Information Engineering of the University of Bologna, Italy. He has published more than 140 papers in international journals or in proceedings of international conferences, and holds two patents. His current research interests are in multiresolution and adaptive signal processing, with a particular emphasis on structural health monitoring applications. 